\title{
Generalized Coupled Fibonacci Sequences
}

\author{
G. P. S. Rathore \\ Department of Mathematics, \\ College of Horticulture, \\ Mandsaur, India
}

\author{
Shweta Jain \\ School of studies in \\ Mathematics, Vikram \\ University, Ujjain, India
}

\author{
Omprakash Sikhwal \\ Department of Mathematics, \\ Mandsaur Institute of \\ Technology, Mandsaur, India
}

\begin{abstract}
In the recent years, there has been much interest in development of knowledge in the general region of Fibonacci numbers and related mathematical topics. The concept of coupled Fibonacci sequences was first introduced by Atanassov, K. T. in 1985. Generalized coupled Fibonacci sequences are defined by

$\alpha_{n}=p \alpha_{n-1}+q \alpha_{n-2}, n \geq 2$ and $\beta_{n}=r \beta_{n-1}+s \beta_{n-2}, n \geq 2$

with initial conditions $\alpha_{0}=a, \alpha_{1}=b, \beta_{0}=c, \beta_{1}=d$.

In this paper, identities of generalized coupled Fibonacci sequences are presented.
\end{abstract}

\section{Subject Classification: 11B39, 11B37}

Keywords: Fibonacci sequence, 2-Fibonacci sequence, generalized coupled Fibonacci sequences.

\section{INTRODUCTION}

The Fibonacci sequence is probably one of the most famous and most widely written about number sequences in all of mathematics. The Fibonacci sequence has been defined by the recurrence relation $F_{n}=F_{n-1}+F_{n-2}, n \geq 2$ with initial conditions $F_{0}=0 \& F_{1}=1$. In which each subsequent filial generation is seen as the sum of the previous two generations. In 1985, Attanasov [1] introduced a new view of a generalized Fibonacci sequences by taking a pair of sequence $\left\{\alpha_{i}\right\}_{i=0}^{\infty}$ and $\left\{\beta_{i}\right\}_{i=0}^{\infty}$, which can be generate by a famous Fibonacci formula and gave various identities involving Fibonacci sequences called them coupled Fibonacci sequences. He was defined and studied about four different ways to generate coupled Fibonacci sequences. $\alpha_{0}=a, \beta_{0}=b, \alpha_{1}=c, \beta_{1}=d$,

$\alpha_{n+2}=\beta_{n+1}+\beta_{n}, \quad n \geq 0$

$\beta_{n+2}=\alpha_{n+1}+\alpha_{n}, \quad n \geq 0$.

$\alpha_{0}=a, \beta_{0}=b, \alpha_{1}=c, \beta_{1}=d$,

$\alpha_{n+2}=\alpha_{n+1}+\beta_{n}, \quad n \geq 0$

$\beta_{n+2}=\beta_{n+1}+\alpha_{n}, \quad n \geq 0$

$\alpha_{0}=a, \beta_{0}=b, \alpha_{1}=c, \beta_{1}=d$,

$\alpha_{n+2}=\beta_{n+1}+\alpha_{n}, \quad n \geq 0$

$\beta_{n+2}=\alpha_{n+1}+\beta_{n}, \quad n \geq 0$.

$\alpha_{0}=a, \beta_{0}=b, \alpha_{1}=c, \beta_{1}=d$,

$\alpha_{n+2}=\alpha_{n+1}+\alpha_{n}, \quad n \geq 0$

$\beta_{n+2}=\beta_{n+1}+\beta_{n}, \quad n \geq 0$.

Singh, M., Sikhwal, O., and Jain, S. [9], present coupled Fibonacci sequences of fifth order with some properties for positive and negative integers. Multiplicative coupled Fibonacci sequences [2] and [4] are deliberated in 1995.
Singh, B. and Sikhwal, O. [10], present fundamental properties of multiplicative coupled Fibonacci sequences of second order. Rathore, G. P. S., Jain, S. and Sikhwal, O. [8], presents multiplicative coupled Fibonacci sequences of third order under two specific schemes. The concept of FibonacciTriple sequences is first introduced by Lee, J. Z., and Lee, J. S., [7] 1987. Singh, B. and Sikhwal, O. [11], presented some fundamental properties of Fibonacci-Triple sequences (3-F sequences). Singh, M., Bhatnagar, S., Sikhwal, O. [12], presented some results on multiplicative triple Fibonacci sequences under two specific schemes.

In this paper, some new identities of generalized coupled Fibonacci sequences are presented.

\section{GENERLIZED COUPLED FIBONACCI SEQUENCES}

Atanassov, K. T. was introduced new generalized coupled Fibonacci sequences. Let $\left\{\alpha_{i}\right\}_{i=0}^{\infty}$ and $\left\{\beta_{i}\right\}_{i=0}^{\infty}$ be two infinite sequences with initial conditions

$\alpha_{0}=a, \alpha_{1}=b, \beta_{0}=c, \beta_{1}=d$, then generalized coupled Fibonacci sequences are defined by

$\alpha_{n}=p \alpha_{n-1}+q \alpha_{n-2}, n \geq 2$

$\beta_{n}=r \beta_{n-1}+s \beta_{n-2}, n \geq 2$

Where $p, q, r$ and $s$ are real numbers

First few terms of the sequences are given below:

\begin{tabular}{|c|c|c|}
\hline$n$ & $\alpha_{n}$ & $\beta_{n}$ \\
\hline 0 & $\mathrm{a}$ & $\mathrm{b}$ \\
\hline 1 & $\mathrm{c}$ & $\mathrm{d}$ \\
\hline 2 & $p c+q a$ & $r d+s b$ \\
\hline 3 & $p^{2} c+p q a+q c$ & $r^{2} d+r s b+s d$ \\
\hline 4 & $p^{3} c+p^{2} q a+2 p q c+q^{2} a$ & $r^{3} d+r^{2} s b+2 r s d+s^{2} b$ \\
\hline
\end{tabular}

\section{MAIN RESULTS}

In this section, some new identities of generalized coupled Fibonacci sequences 2.1 will be discussed. Many authors have been presented identities of coupled Fibonacci sequences under additive and multiplicative patterns. In this section, some sum formulae of $\mathrm{n}$ terms of coupled Fibonacci sequences are presented

Theorem (3.1): Sum of the first $n$ terms of generalized coupled Fibonacci sequence is 
$(p+q-1)\left(\alpha_{1}+\alpha_{2}+\alpha_{3}+\ldots+\alpha_{n}\right)+(r+s-1)\left(\beta_{1}+\beta_{2}+\beta_{3}+\ldots+\beta_{n}\right)$

$=\alpha_{n+1}+q \alpha_{n}+\beta_{n+1}+s \beta_{n}-q a-s b-c-d$.

Proof: By (2.1), to obtain

$q \alpha_{1}+s \beta_{1}=\alpha_{3}+\beta_{3}-p \alpha_{2}-r \beta_{2}$, $q \alpha_{2}+s \beta_{2}=\alpha_{4}+\beta_{4}-p \alpha_{3}-r \beta_{3}$, $q \alpha_{3}+s \beta_{3}=\alpha_{5}+\beta_{5}-p \alpha_{4}-r \beta_{4}$,

$q \alpha_{n-1}+s \beta_{n-1}=\alpha_{n+1}+\beta_{n+1}-p \alpha_{n}-r \beta_{n}$, $q \alpha_{n}+s \beta_{n}=\alpha_{n+2}+\beta_{n+2}-p \alpha_{n+1}-r \beta_{n+1}$.

Term wise addition of all above equations,

$$
\begin{aligned}
& q\left(\alpha_{1}+\alpha_{2}+\alpha_{3}+\ldots+\alpha_{n}\right)+s\left(\beta_{1}+\beta_{2}+\beta_{3}+\ldots+\beta_{n}\right) \\
& =\left(\alpha_{3}+\alpha_{4}+\alpha_{5}+\ldots+\alpha_{n+2}\right)+\left(\beta_{3}+\beta_{4}+\beta_{5}+\ldots+\beta_{n+2}\right) \\
& -p\left(\alpha_{2}+\alpha_{3}+\ldots+\alpha_{n+1}\right)-r\left(\beta_{2}+\beta_{3}+\ldots+\beta_{n+1}\right) \\
& q\left(\alpha_{1}+\alpha_{2}+\alpha_{3}+\ldots+\alpha_{n}\right)+s\left(\beta_{1}+\beta_{2}+\beta_{3}+\ldots+\beta_{n}\right) \\
& =\left(\alpha_{3}+\alpha_{4}+\alpha_{5}+\ldots+\alpha_{n+2}\right)+\left(\beta_{3}+\beta_{4}+\beta_{5}+\ldots+\beta_{n+2}\right) \\
& +\alpha_{1}+\alpha_{2}-\alpha_{1}-\alpha_{2}+\beta_{1}+\beta_{2}-\beta_{1}-\beta_{2}-p\left(\alpha_{2}+\alpha_{3}+\ldots\right. \\
& \left.+\alpha_{n+1}+\alpha_{1}-\alpha_{1}\right)-r\left(\beta_{2}+\beta_{3}+\ldots+\beta_{n+1}+\beta_{1}-\beta_{1}\right) \\
& q\left(\alpha_{1}+\alpha_{2}+\alpha_{3}+\ldots+\alpha_{n}\right)+s\left(\beta_{1}+\beta_{2}+\beta_{3}+\ldots+\beta_{n}\right) \\
& =\left(\alpha_{1}+\alpha_{2}+\alpha_{3}+\ldots+\alpha_{n}\right)+\left(\beta_{1}+\beta_{2}+\beta_{3}+\ldots+\beta_{n}\right) \\
& -p\left(\alpha_{1}+\alpha_{2}+\alpha_{3}+\ldots+\alpha_{n}\right)-r\left(\beta_{1}+\beta_{2}+\beta_{3}+\ldots+\beta_{n}\right) \\
& +\alpha_{n+1}+\alpha_{n+2}+\beta_{n+1}+\beta_{n+2}-\alpha_{1}-\alpha_{2}-\beta_{1}-\beta_{2} \\
& +p \alpha_{1}-p \alpha_{n+1}+r \beta_{1}-r \beta_{n+1}
\end{aligned}
$$

$(p+q-1)\left(\alpha_{1}+\alpha_{2}+\alpha_{3}+\ldots+\alpha_{n}\right)+(r+s-1)\left(\beta_{1}+\beta_{2}+\beta_{3}+\ldots+\beta_{n}\right)$ $=(1-p) \alpha_{n+1}+(1-r) \beta_{n+1}+\alpha_{n+2}-\alpha_{2}+\beta_{n+2}-\beta_{2}+(p-1) \alpha_{1}+(r-1) \beta_{1}$

$(p+q-1)\left(\alpha_{1}+\alpha_{2}+\alpha_{3}+\ldots+\alpha_{n}\right)+(r+s-1)\left(\beta_{1}+\beta_{2}+\beta_{3}+\ldots+\beta_{n}\right)$ $=(1-p) \alpha_{n+1}+(1-r) \beta_{n+1}+(p-1) \alpha_{1}+(r-1) \beta_{1}+p \alpha_{n+1}+q \alpha_{n}-\alpha_{2}$ $+r \beta_{n+1}+s \beta_{n}-\beta_{2}$

$(p+q-1)\left(\alpha_{1}+\alpha_{2}+\alpha_{3}+\ldots+\alpha_{n}\right)+(r+s-1)\left(\beta_{1}+\beta_{2}+\beta_{3}+\ldots+\beta_{n}\right)$ $=\alpha_{n+1}+\beta_{n+1}+q \alpha_{n}+s \beta_{n}-p c-q a-r d-s b+p c+r d-c-d$

$(p+q-1)\left(\alpha_{1}+\alpha_{2}+\alpha_{3}+\ldots+\alpha_{n}\right)+(r+s-1)\left(\beta_{1}+\beta_{2}+\beta_{3}+\ldots+\beta_{n}\right)$ $=\alpha_{n+1}+q \alpha_{n}+\beta_{n+1}+s \beta_{n}-q a-s b-c-d$.

Theorem (3.2): Sum of the first $n$ terms with odd indices is

$$
\begin{aligned}
& \left(q^{n} \alpha_{1}+q^{n-1} \alpha_{3}+q^{n-2} \alpha_{5}+\ldots+q \alpha_{2 n-1}\right)+\left(s^{n} \beta_{1}+s^{n-1} \beta_{3}+s^{n-2} \beta_{5}+\ldots+s \beta_{2 n-1}\right) \\
& =\frac{q}{p}\left(\alpha_{2 n}-q^{n} a\right)+\frac{s}{r}\left(\beta_{2 n}-s^{n} b\right) .
\end{aligned}
$$

Proof: By (2.1), to obtain

$$
\begin{aligned}
& \alpha_{1}+\beta_{1}=\frac{\alpha_{2}-q \alpha_{0}}{p}+\frac{\beta_{2}-s \beta_{0}}{r}, \\
& \alpha_{3}+\beta_{3}=\frac{\alpha_{4}-q \alpha_{2}}{p}+\frac{\beta_{4}-s \beta_{2}}{r}, \\
& \alpha_{5}+\beta_{5}=\frac{\alpha_{6}-q \alpha_{4}}{p}+\frac{\beta_{6}-s \beta_{4}}{r},
\end{aligned}
$$

... $\cdots \cdots$

$\alpha_{2 n-1}+\beta_{2 n-1}=\frac{\alpha_{2 n}-q \alpha_{2 n-2}}{p}+\frac{\beta_{2 n-}-s \beta_{2 n-2}}{r}$.

Multiplying $\alpha_{1}, \alpha_{3}, \alpha_{5} \ldots \alpha_{2 n-1}$ by $q^{n}, q^{n-1}, q^{n-2}, \ldots, q^{2}, q$

$$
\& \beta_{1}, \beta_{3}, \beta_{5} \ldots \beta_{2 n-1} \text { by } s^{n}, s^{n-1}, s^{n-2}, \ldots, s^{2}, s
$$

Respectively and adding, to obtain

$$
\begin{aligned}
& q^{n} \alpha_{1}+s^{n} \beta_{1}+q^{n-1} \alpha_{3}+s^{n-1} \beta_{3}+q^{n-2} \alpha_{5}+s^{n-2} \beta_{5} \ldots+q \alpha_{2 n-1}+s \beta_{2 n-1} \\
& =q^{n}\left(\frac{\alpha_{2}-q \alpha_{0}}{p}\right)+s^{n}\left(\frac{\beta_{2}-s \beta_{0}}{r}\right)+q^{n-1}\left(\frac{\alpha_{4}-q \alpha_{2}}{p}\right)+s^{n-1}\left(\frac{\beta_{4}-s \beta_{2}}{r}\right) \\
& +q^{n-2}\left(\frac{\alpha_{6}-q \alpha_{4}}{p}\right)+s^{n-1}\left(\frac{\beta_{6}-s \beta_{4}}{r}\right)+\ldots+q\left(\frac{\alpha_{2 n}-q \alpha_{2 n-2}}{p}\right)+s\left(\frac{\beta_{2 n-}-s \beta_{2 n-2}}{r}\right)
\end{aligned}
$$

$\left(q^{n} \alpha_{1}+q^{n-1} \alpha_{3}+q^{n-2} \alpha_{5}+\ldots+q \alpha_{2 n-1}\right)+\left(s^{n} \beta_{1}+s^{n-1} \beta_{3}+s^{n-2} \beta_{5}+\ldots+s \beta_{2 n-1}\right)$

$=\frac{q}{p}\left(\alpha_{2 n}-q^{n} \alpha_{0}\right)+\frac{s}{r}\left(\beta_{2 n}-s^{n} \beta_{0}\right)$

$\left(q^{n} \alpha_{1}+q^{n-1} \alpha_{3}+q^{n-2} \alpha_{5}+\ldots+q \alpha_{2 n-1}\right)+\left(s^{n} \beta_{1}+s^{n-1} \beta_{3}+s^{n-2} \beta_{5}+\ldots+s \beta_{2 n-1}\right)$

$=\frac{q}{p}\left(\alpha_{2 n}-q^{n} a\right)+\frac{s}{r}\left(\beta_{2 n}-s^{n} b\right)$.

Theorem (3.3): Sum of the first $n$ terms with even indices is $\left(q^{n} \alpha_{2}+q^{n-1} \alpha_{4}+q^{n-2} \alpha_{6}+\ldots+q \alpha_{2 n}\right)+\left(s^{n} \beta_{2}+s^{n-1} \beta_{4}+s^{n-2} \beta_{6}+\ldots+s \beta_{2 n}\right)$ $=\frac{q}{p}\left(\alpha_{2 n+1}-q^{n} c\right)+\frac{s}{r}\left(\beta_{2 n+1}-s^{n} d\right)$.

Proof: By (2.1), to obtain

$$
\begin{aligned}
& \alpha_{2}+\beta_{2}=\frac{\alpha_{3}-q \alpha_{1}}{p}+\frac{\beta_{3}-s \beta_{1}}{r}, \\
& \alpha_{4}+\beta_{4}=\frac{\alpha_{5}-q \alpha_{3}}{p}+\frac{\beta_{5}-s \beta_{3}}{r}, \\
& \alpha_{6}+\beta_{6}=\frac{\alpha_{7}-q \alpha_{5}}{p}+\frac{\beta_{7}-s \beta_{5}}{r}, \\
& \ldots \ldots \ldots \\
& \alpha_{2 n}+\beta_{2 n}=\frac{\alpha_{2 n+1}-q \alpha_{2 n-1}}{p}+\frac{\beta_{2 n+1}-s \beta_{2 n-1}}{r} .
\end{aligned}
$$


Multiplying $\alpha_{2}, \alpha_{4}, \alpha_{6} \ldots \alpha_{2 n}$ by $q^{n}, q^{n-1}, q^{n-2}, \ldots, q^{2}, q$ $\& \beta_{2}, \beta_{4}, \beta_{6} \ldots \beta_{2 n}$ by $s^{n}, s^{n-1}, s^{n-2}, \ldots, s^{2}, s$ respectively and adding, to obtain

$$
\begin{aligned}
& q^{n} \alpha_{2}+s^{n} \beta_{2}+q^{n-1} \alpha_{4}+s^{n-1} \beta_{4}+q^{n-2} \alpha_{6}+s^{n-2} \beta_{6} \ldots+q \alpha_{2 n}+s \beta_{2 n} \\
& =q^{n}\left(\frac{\alpha_{3}-q \alpha_{1}}{p}\right)+s^{n}\left(\frac{\beta_{3}-s \beta_{1}}{r}\right)+q^{n-1}\left(\frac{\alpha_{5}-q \alpha_{3}}{p}\right)+s^{n-1}\left(\frac{\beta_{5}-s \beta_{3}}{r}\right) \\
& +q^{n-2}\left(\frac{\alpha_{7}-q \alpha_{5}}{p}\right)+s^{n-1}\left(\frac{\beta_{7}-s \beta_{5}}{r}\right)+\ldots+q\left(\frac{\alpha_{2 n+1}-q \alpha_{2 n-1}}{p}\right)+s\left(\frac{\beta_{2 n+1}-s \beta_{2 n-1}}{r}\right) . \\
& \left(q^{n} \alpha_{2}+q^{n-1} \alpha_{4}+q^{n-2} \alpha_{6}+\ldots+q \alpha_{2 n}\right)+\left(s^{n} \beta_{2}+s^{n-1} \beta_{4}+s^{n-2} \beta_{6}+\ldots+s \beta_{2 n}\right) \\
& =\frac{q}{p}\left(\alpha_{2 n+1}-q^{n} \alpha_{1}\right)+\frac{s}{r}\left(\beta_{2 n+1}-s^{n} \beta_{1}\right) \\
& \left(q^{n} \alpha_{2}+q^{n-1} \alpha_{4}+q^{n-2} \alpha_{6}+\ldots+q \alpha_{2 n}\right)+\left(s^{n} \beta_{2}+s^{n-1} \beta_{4}+s^{n-2} \beta_{6}+\ldots+s \beta_{2 n}\right) \\
& =\frac{q}{p}\left(\alpha_{2 n+1}-q^{n} c\right)+\frac{s}{r}\left(\beta_{2 n+1}-s^{n} d\right) .
\end{aligned}
$$

Theorem (3.4): For positive integer $n$,

$p \alpha_{n}^{2}+s \beta_{n}^{2}=\alpha_{n} \alpha_{n+1}-q \alpha_{n-1} \alpha_{n}+\beta_{n} \beta_{n+1}-s \beta_{n-1} \beta_{n}$.

Proof: $\alpha_{n} \alpha_{n+1}-q \alpha_{n-1} \alpha_{n}+\beta_{n} \beta_{n+1}-s \beta_{n-1} \beta_{n}$ $=\alpha_{n}\left(\alpha_{n+1}-q \alpha_{n-1}\right)+\beta_{n}\left(\beta_{n+1}-s \beta_{n-1}\right)$

$\alpha_{n} \alpha_{n+1}-q \alpha_{n-1} \alpha_{n}+\beta_{n} \beta_{n+1}-s \beta_{n-1} \beta_{n}$

$=\alpha_{n}\left(p \alpha_{n}\right)+\beta_{n}\left(r \beta_{n}\right)$

$\alpha_{n} \alpha_{n+1}-q \alpha_{n-1} \alpha_{n}+\beta_{n} \beta_{n+1}-s \beta_{n-1} \beta_{n}=p \alpha_{n}^{2}+s \beta_{n}^{2}$.

Theorem (3.5): Sum of the square of first $n$ terms is

$p\left(q^{n-1} \alpha_{1}^{2}+q^{n-2} \alpha_{2}^{2}+q^{n-3} \alpha_{3}^{2}+\ldots+q \alpha_{n-1}^{2}+\alpha_{n}^{2}\right)+$

$\left(r s^{n-1} \beta_{1}^{2}+s^{n-2} \beta_{2}^{2}+s^{n-3} \beta_{3}^{2}+\ldots+q \beta_{n-1}^{2}+\beta_{n}^{2}\right)$

$=\alpha_{n} \alpha_{n+1}-q^{n} a c+\beta_{n} \beta_{n+1}-s^{n} b d$.

Proof: By Theorem (3.4), we have

$p \alpha_{1}^{2}+r \beta_{1}^{2}=\alpha_{1} \alpha_{2}-q \alpha_{0} \alpha_{1}+\beta_{1} \beta_{2}-s \beta_{0} \beta_{1}$,

$p \alpha_{2}^{2}+r \beta_{2}^{2}=\alpha_{2} \alpha_{3}-q \alpha_{1} \alpha_{2}+\beta_{2} \beta_{3}-s \beta_{1} \beta_{2}$,

$p \alpha_{3}^{2}+r \beta_{3}^{2}=\alpha_{3} \alpha_{4}-q \alpha_{2} \alpha_{3}+\beta_{3} \beta_{4}-s \beta_{2} \beta_{3}$,

$p \alpha_{n}^{2}+r \beta_{n}^{2}=\alpha_{n} \alpha_{n+1}-q \alpha_{n-1} \alpha_{n}+\beta_{n} \beta_{n+1}-s \beta_{n-1} \beta_{n}$,

Multiplying $p \alpha_{1}^{2}, p \alpha_{2}^{2} \ldots p \alpha_{n}^{2}$ by $q^{n-1}, q^{n-2}, \ldots, q, 1$

$$
\& r \beta_{1}^{2}, r \beta_{2}^{2} \ldots r \beta_{n}^{2} \text { by } s^{n-1}, s^{n-2}, \ldots, s, 1
$$

respectively and adding, to obtain

$\left(q^{n-1} p \alpha_{1}^{2}+q^{n-2} p \alpha_{2}^{2}+\ldots+p \alpha_{n}^{2}\right)+\left(s^{n-1} r \beta_{1}^{2}+s^{n-2} r \beta_{2}^{2}+\ldots+s r \beta_{n}^{2}\right)$

$=q^{n-1}\left(\alpha_{1} \alpha_{2}-q \alpha_{0} \alpha_{1}\right)+s^{n-1}\left(\beta_{1} \beta_{2}-s \beta_{0} \beta_{1}\right)+q^{n-2}\left(\alpha_{2} \alpha_{3}-q \alpha_{1} \alpha_{2}\right)$

$+s^{n-1}\left(\beta_{2} \beta_{3}-s \beta_{1} \beta_{2}\right)+\ldots+\alpha_{n} \alpha_{n+1}-q \alpha_{n-1} \alpha_{n}+\beta_{n} \beta_{n+1}-s \beta_{n-1} \beta_{n}$,

$$
\begin{aligned}
& p\left(q^{n-1} \alpha_{1}^{2}+q^{n-2} \alpha_{2}^{2}+\ldots+\alpha_{n}^{2}\right)+r\left(s^{n-1} \beta_{1}^{2}+s^{n-2} \beta_{2}^{2}+\ldots+s \beta_{n}^{2}\right) \\
& =\alpha_{n} \alpha_{n+1}-q^{n} \alpha_{0} \alpha_{1}+\beta_{n} \beta_{n+1}-s^{n} \beta_{0} \beta_{1} \\
& p\left(q^{n-1} \alpha_{1}^{2}+q^{n-2} \alpha_{2}^{2}+q^{n-3} \alpha_{3}^{2}+\ldots+q \alpha_{n-1}^{2}+\alpha_{n}^{2}\right)+ \\
& \left(r s^{n-1} \beta_{1}^{2}+s^{n-2} \beta_{2}^{2}+s^{n-3} \beta_{3}^{2}+\ldots+q \beta_{n-1}^{2}+\beta_{n}^{2}\right) \\
& =\alpha_{n} \alpha_{n+1}-q^{n} a c+\beta_{n} \beta_{n+1}-s^{n} b d .
\end{aligned}
$$

\section{CONCLUSION}

In this paper, identities of generalized coupled Fibonacci sequences are presented. For values of $p, q, r$ and $s$, the identities of classical coupled Fibonacci sequences can be obtained. In future, identities of coupled Fibonacci sequences of higher order can be formed under additive and multiplicative patterns. Also identities can be formed for Fibonacci-Triple Sequences under additive and multiplicative patterns.

\section{ACKNOWLEDGMENTS}

Authors are thankful to reviewers for their valuable comments in improvement of the paper.

\section{REFERENCES}

[1] Atanassov K. T., 1986. On a Second New Generalization Of the Fibonacci Sequence, The Fibonacci Quarterly, Vol. 24, No. 4, 362-365.

[2] Atanassov K. T., 1995. Remark on a New Direction for a Generalization of the Fibonacci Sequence, The Fibonacci Quarterly, Vol. 33, No. 3, 249-250.

[3] Atanassov K. T., Atanassov L. C. and Sasselov D. D., 1985. A New Perspective to the Generalization of the Fibonacci sequence, The Fibonacci Quarterly, Vol. 23, No. 1, 21-28.

[4] Atanassov K T., Atanassov V., Shannon A.and Turner J., 2002. New Visual perspective On Fibonacci number, World Scientific Publishing Company, Singapore.

[5] Hordam, A. F., 1965. Basic properties of certain Generalized sequence of numbers, The Fibonacci Quarterly, 3, 161-176.

[6] Hordam, A. F., 1961. The generalized Fibonacci Sequences, the American Math. Monthly, Vol. 68, No. 5 455-459.

[7] Lee J. Z. and Lee J. S., 1987. Some Properties of the Generalization of the Fibonacci sequence, The Fibonacci Quarterly, Vol. 25, No. 2, 111-117.

[8] Rathore G.P.S., Jain. S., Sikhwal O., 2012. Multiplicative Coupled Fibonacci Sequences of third order, Int. J. Contemp. Math. Sciences, Vol. 7, No. 31, 1535 - 1540.

[9] Singh M., Sikhwal O. and Jain S., 2010. Coupled Fibonacci Sequences of Fifth Order and Some Properties International Journal of Mathematical Analysis, Vol. 4, No. 25, 1247-1254, Bulgaria.

[10] Singh B. and Sikhwal O., 2010. Multiplicative coupled Fibonacci sequences and some fundamental properties, International journals contemporary mathematical Sciences, Vol. 5, No. 5, 223-230. 
[11] Singh B. and Sikhwal O., Fibonacci-triple sequences and Some fundamental properties, Tamkang Journal ofMathematics, Vol. 41, No. 4 (2010), 325-333.

[12] Singh, M., Bhatnagar, S., Sikhwal, O., 2011, Multiplicative Triple Fibonacci sequences, Applied Mathematical Sciences, Vol. 6, No. 52, 2567 - 2572
[13]Sikhwal O., 2012 Generalization of Fibonacci sequence: An Intriguing Sequence, Lap Lambert Academic Publishing GmbH \& Co. KG, Germany. 